\title{
Current bursts in lipid bilayers initiated by colloidal quantum dots
}

\author{
Sujatha Ramachandran, George L. Kumar, Robert H. Blick, ${ }^{a)}$ and Daniel W. van der Weide \\ LMSE-Laboratory for Molecular Scale Engineering, Electrical and Computer Engineering, \\ University of Wisconsin-Madison, Wisconsin 53706
}

(Received 13 August 2004; accepted 20 December 2004; published online 17 February 2005)

Inorganic semiconductor nanocrystals, also called quantum dots, have recently attracted considerable interest as fluorescent labels. We report that CdSe QDs initiate current bursts in lipid bilayer membranes upon application of a bias voltage. The current bursts observed resemble those produced by the peptaibol class of antibiotics such as alamethicin and trichorzins. The current fluctuations are dependent on the bias voltage and on the concentration of the quantum dots applied to the membrane. Our data suggest that quantum dots with dipole moments similar to alamethicin are influenced by an external electric field, which creates a torque resulting in the insertion into the lipid membrane. We predict that at least three quantum dots are required to form a pore due to aggregation that leads to a macroscopic conductance. (C) 2005 American Institute of Physics.

[DOI: $10.1063 / 1.1862752]$

Inorganic semiconductor nanocrystals, also called colloidal quantum dots (QDs), are useful fluorescent labels for staining cells. Because of their size dependent color, their resistance to photobleaching, and size scale of less than $10 \mathrm{~nm}$, they are becoming popular in many interesting biomedical applications ranging from intracellular tagging of biological molecules to applications such as tracking devices for neuronal receptors and as interfaces between nerve cells. ${ }^{1,2}$ Because many of the applications of QDs in cell biology involve interaction with lipid bilayer membranes, the behavior of QDs on these structures is of significant interest. We chose planar lipid bilayers as our study material since they form the basis of a simple model system for eukaryotic cell membranes where many ion channel and receptor activity studies have been conducted. ${ }^{3}$ A planar lipid bilayer is about $5 \mathrm{~nm}$ thick and composed of two layers of selfassembled amphiphilic lipid molecules such as phospholipids arranged so that their hydrocarbon tails face one another to form the hydrophobic core while their electrically charged polar heads face the aqueous solution on either side of the membrane [Fig. 1(a)].

In this letter, we study the behavior of colloidal QDs in planar lipid bilayers as indicated in Fig. 1(a) using electrical recording techniques. ${ }^{4}$ The QDs used in this study were colloidal CdSe nanocrystals. Three different QDs were used: (a) CdSe cores in toluene (Evident Technologies, Troy, NY), (b) Custom-made water-soluble CdSe cores with a $\mathrm{ZnS}$ shell coated with a polymer (Evident Technologies, Troy, NY), and (c) water-soluble streptavidin-conjugated $\mathrm{CdSe}$ cores with ZnS shell (Quantum Dot Corporation, Hayward, CA). To study their size QDs were deposited onto formvar free carbon film supported by a 300 mesh copper grid (Ted Pella, Inc., Redding, CA) and examined using a Philips CM120 transmission electron microscope operated at an accelerating voltage of $60 \mathrm{kV}(300000 \times)$. The average sizes of the QDs were: core CdSe QDs $=(2.2 \pm 0.5) \mathrm{nm}$, water-soluble polymer coated $\mathrm{CdSe} / \mathrm{ZnS}$ QDs $=(3.2 \pm 0.5) \mathrm{nm}$, and the core/shell di-

\footnotetext{
a) Author to whom correspondence should be addressed; electronic mail:
} blick@engr.wisc.edu ameter of water-soluble streptavidin-conjugated QDs $=(5.7 \pm 0.8) \mathrm{nm}$.

Planar lipid bilayer membranes were formed from 1, 2-diphytanoyl phosphatidylcholine (DiPhyPC) in decane (Avanti Polar Lipids, Inc., Alabaster, AL) across a 200$\mu \mathrm{m}$-diam orifice in a Delrin cuvette (Warner Instruments, Inc., Hamden, CT), separating two aqueous compartments containing $1 \mathrm{ml}$ of $1 \mathrm{M} \mathrm{KCl}, 10 \mathrm{mM}$ HEPES, at $p \mathrm{H} 7.5$

(a)

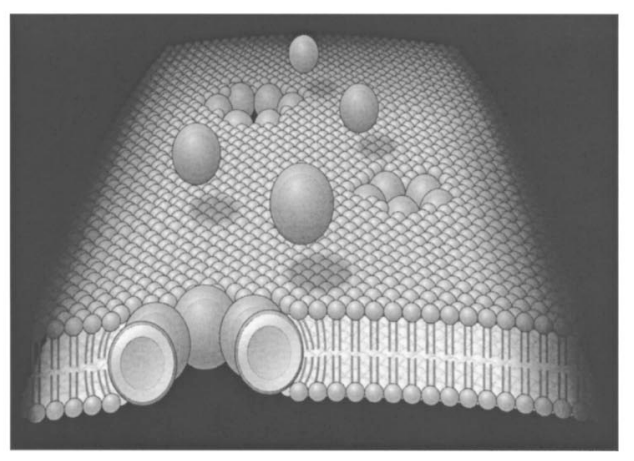

(b)

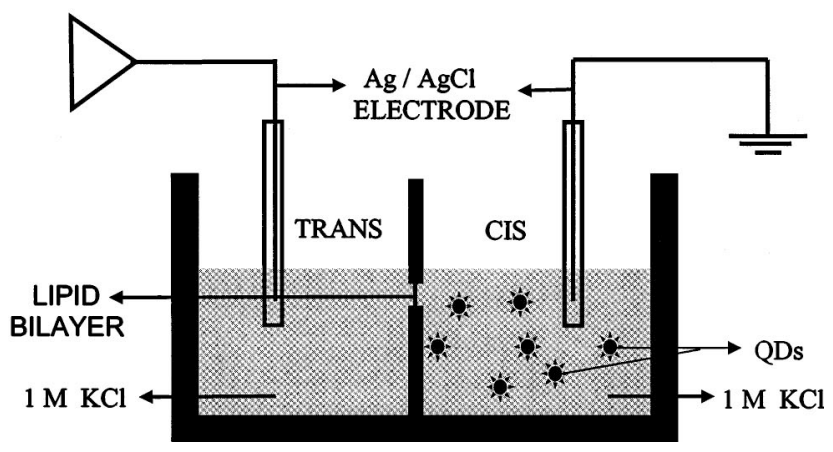

FIG. 1. (a) Suggested mechanism of QD insertion into the lipid bilayer membrane. When a membrane is polarized by an applied potential, QDs possessing a dipole moment insert into the membrane and form pores. (b) Schematic diagram of the measurement setup. 

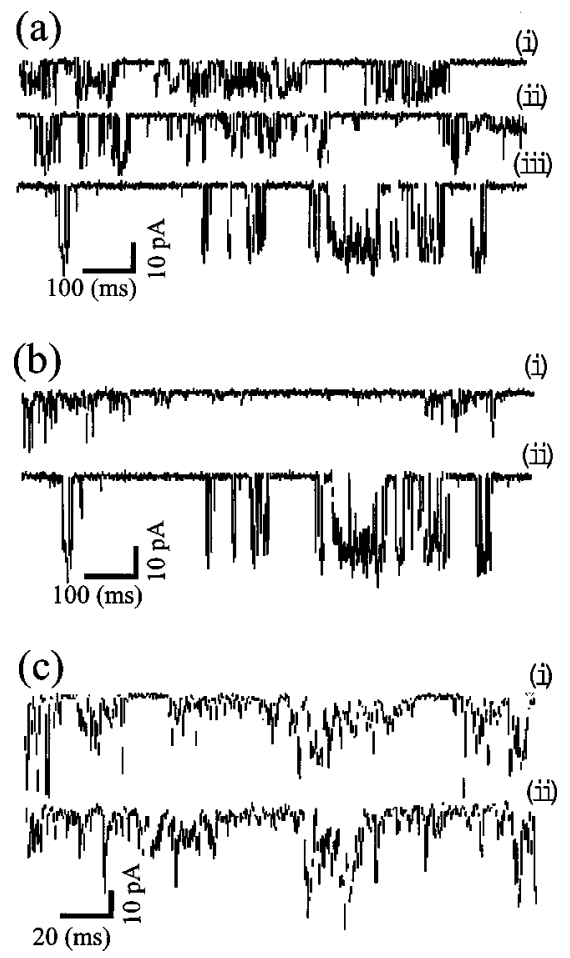

FIG. 2. Current fluctuations measured across the lipid bilayer in the presence of QDs. (a) Voltage-dependent current bursts in the presence of streptavidin-conjugated QDs at a concentration of $6 \mathrm{nM}$ in a buffer containing $1 \mathrm{M} \mathrm{KCl}, 10 \mathrm{mM}$ HEPES $p \mathrm{H}$ 7.5. Bias voltages are (i-iii) $-30,-40$, and $-50 \mathrm{mV}$, respectively. (b) Current fluctuations under two different concentrations of streptavidin-conjugated QDs at a bias of $-50 \mathrm{mV}$ : (i) at $3 \mathrm{nM}$ and (ii) at $6 \mathrm{nM}$. (c) Current bursts for (i-ii) 2.2 and $3.2 \mathrm{~nm}$ QDs, respectively, at $-50 \mathrm{mV}$ bias.

$\left(23^{\circ} \mathrm{C}\right)$. A schematic diagram of the measurement setup is shown in Fig. 1(b). The compartments were connected to an integrating patch clamp amplifier (Axopatch $200 \mathrm{~B}$, Axon Instruments, Inc., Union City, CA) via a pair of $\mathrm{Ag} / \mathrm{AgCl}$ electrodes in $0.5 \mathrm{M} \mathrm{KCl}, 1 \%$ agar bridges. QDs of varying concentrations were added to the cis side of the chamber. The trans side was connected to the head stage of the amplifier, and the cis compartment was held at virtual ground. Resulting currents were low-pass filtered with a built-in fourpole Bessel filter at $1 \mathrm{kHz}$ and sampled at $10 \mathrm{kHz}$ by computer with a Digidata 1320 analog-to-digital converter. As a control, $5 \mathrm{~nm}$ streptavidin-conjugated gold particles (Ted Pella. Inc., Redding, CA), were added to the cis side to a final concentration of $3.4 \times 10^{11}$ gold particles $/ \mathrm{ml}$ to study their effects on the lipid bilayer in the presence of voltage bias ranging from 0 to $200 \mathrm{mV}$. No current was recorded for the gold particles.

Figure 2 represents the traces of current fluctuations measured across the lipid bilayer in the presence of the three different QDs used in our experiment. As shown in Fig. 2(a), we found a voltage dependent current flow through the membrane in the presence of streptavidin-conjugated $\mathrm{CdSe} / \mathrm{ZnS}$ QDs on application of a negative voltage. Also, increasing the concentration of the QDs resulted in increased conductance [Fig. 2(b)]. Similar current bursts across the lipid bilayer were observed with the core CdSe and polymer-coated CdSe/ZnS QDs [Fig. 2(c)]. Although the current exhibits a stochastic pattern without discrete levels, the effect is repeatable at a given voltage and QD concentration. Figure 3 shows the current burst frequency distribution histograms Downloaded 01 Dec 2006 to 128.104.198.120. Redistribution subject

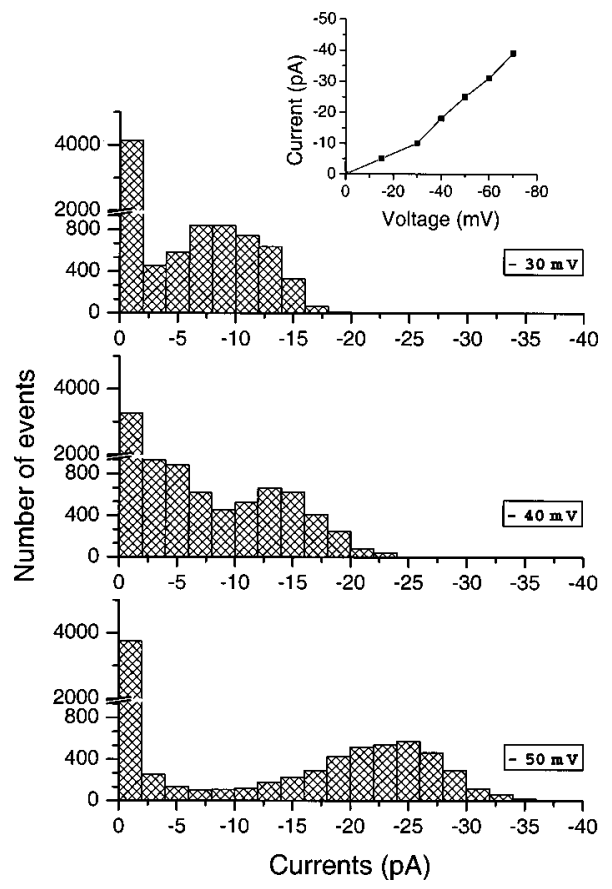

FIG. 3. Current burst frequency distribution histogram of streptavidinconjugated QDs at varying voltage. Inset shows a current/voltage plot for the streptavidin-conjugated QDs.

and a current/voltage plot showing the ohmic nature of the current burst (plotted in the inset). Furthermore, we observed that reverse voltage bias did not elicit current bursts [Fig. 4(a)]. A control experiment using $5 \mathrm{~nm}$ streptavidinconjugated gold particles failed to elicit current fluctuations [Fig. 4(b)].

Our data suggest: (I) QDs do not diffuse readily through the dielectric bilayer membrane, because the lipid bilayer forms a barrier to diffusion of ions and polar molecules larger than about 150 Daltons. (II) The current bursts we observe with QDs are very similar to alamethicin, a polypeptide antibiotic, which aggregates and forms voltagedependent ion conducting pores in lipid membranes. ${ }^{5}$ It has been suggested that the alamethicin monomer is about $3.5 \mathrm{~nm}$ in length and carries a dipole moment of approximately $75 \mathrm{D}$ units, which is responsible for the voltage-

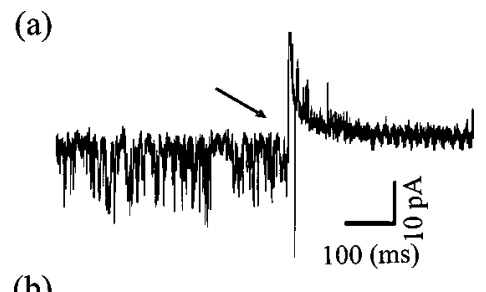

(b)

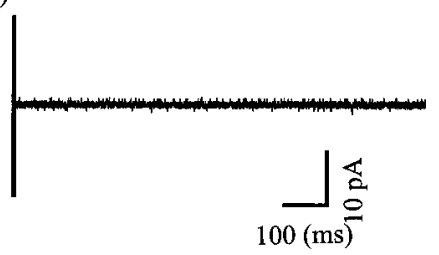

FIG. 4. (a) Reverse bias voltage trace of streptavidin-conjugated QDs. Arrow indicates reversing the bias voltage from -40 to $+40 \mathrm{mV}$. No current bursts are seen with positive voltage bias. (b) Current trace in presence of streptavidin-conjugated gold nanoparticles $\left(3.4 \times 10^{11}\right.$ gold particles $\left./ \mathrm{ml}\right)$. Changing the bias in the range from $-200-+200 \mathrm{mV}$ resulted in a similar

zero-current trace.
to AlP license or copyright, see http://apl.aip.org/apl/copyright.jsp 
TABLE I. Number of QDs required to form a pore and their respective pore diameters for a conductance of $500 \mathrm{pS}(25 \mathrm{pA}$ at $50 \mathrm{mV})$. Calculations were made on approximations used in the calculation of alamethicin pore. $^{\mathrm{a}}$

\begin{tabular}{|c|c|c|c|}
\hline & $\begin{array}{l}\text { Diameter of QDs } \\
\quad(\mathrm{nm})\end{array}$ & $\begin{array}{c}\text { Number of QDs required } \\
\text { to form a pore }\end{array}$ & $\begin{array}{l}\text { Diameter of pore } \\
\qquad(\mathrm{nm})\end{array}$ \\
\hline $\mathrm{CdSe}$ & $2.2 \pm 0.5$ & 3.04 & 0.36 \\
\hline $\begin{array}{l}\mathrm{CdSe} / \mathrm{ZnS}+ \\
\text { polymer }\end{array}$ & $3.2 \pm 0.5$ & 2.91 & 0.43 \\
\hline $\begin{array}{c}\mathrm{CdSe} / \mathrm{ZnS}+ \\
\text { polymer+ } \\
\text { streptavidin }\end{array}$ & $10-15^{\mathrm{b}}$ & $\begin{array}{l}2.63 \text { for } 10 \mathrm{~nm} \text { QD } \\
2.57 \text { for } 15 \mathrm{~nm} \text { QD }\end{array}$ & $\begin{array}{l}0.75 \text { for } 10 \mathrm{~nm} \mathrm{QD} \\
0.92 \text { for } 15 \mathrm{~nm} \mathrm{QD}\end{array}$ \\
\hline
\end{tabular}

dependent conductance. ${ }^{6,7}$ We can conclude that the QDs, having a permanent dipole moment ${ }^{8}$ similar to alamethicin, are influenced by an external electric field, which creates a torque on the QD dipole, forcing insertion into the lipid membrane [Fig. 1(a)]. (III) Because many voltage-dependent current bursts are observed in $1 \mathrm{~s}$ sometimes spaced a few milliseconds apart, it is possible that oligomeric aggregation of QDs forms pores leading to the macroscopic conductance we observe. In addition, we observe smaller current peaks that could be caused by single QDs embedded in the lipid membrane, which could cause a voltage-dependent flip-flop of lipids disrupting the membrane. We believe that the opening and closing of the channel occurs by random association and dissociation of QDs caused by disruptive forces of thermal agitation, and several other factors including surface tension of the lipid bilayer. Unlike peptide ion channels, which have defined structures and are attached to the membranes more stably, the QDs will only be weakly associated. This causes instability, and thus a nonstationary current flow is detected.

To determine both the "pore" size required for the macroscopic conductance observed and the number of QDs forming a pore [see Fig. 1(a)], we measured the conductivity of $1 \mathrm{M} \mathrm{KCl}$ at $23{ }^{\circ} \mathrm{C}$, and found it to be 11.10 $\times 10^{4} \mu \mathrm{S} / \mathrm{cm}$. For an applied bias voltage of $50 \mathrm{mV}$ and a current of $25 \mathrm{pA}$ (for a large pore), we get a conductance of $500 \mathrm{pS}$. Assuming we have a very large pore in an uncharged membrane so that its conductivity is equal to that of $1 \mathrm{M}$ $\mathrm{KCl}$, we find the pore diameter responsible for a $500 \mathrm{pS}$ conductance using $\sigma=G \times h / A$. Here, $\sigma$ is the conductivity of bulk solution; $G$ is the conductance; $h$ is the height of the QD (=diameter of the QD as determined from electron microscopy), and $A$ is the area $\left(=\pi r^{2}\right.$, where $r=$ pore radius $)$ to get pore diameters $\left(d_{\text {pore }}\right)$ of $0.36,0.43$, and $0.75-0.92 \mathrm{~nm}$ formed by the smallest to largest sizes of QDs, respectively (Table I). Based on electron microscopy data, and on the assumption that QDs are perfect spheres coming close together to form a pore, we find the number of QDs required to form a pore using the relation $d_{\text {pore }}=d_{\text {dot }}(1 / \sin (\pi / N)-1)$ with $N$ being the number of QDs, $d_{\text {pore }}=$ diameter of the pore formed by QDs and $d_{\text {dot }}=$ diameter of the QD. We predict that a conductance of $500 \mathrm{pS}$ results from three QDs assembling to form an electrolyte filled pore with diameters 0.36 , 0.43 , and $0.75-0.92 \mathrm{~nm}$, depending on the diameters of the QDs used (see Table I). The values of pore sizes we calculate correspond closely to the values that have been calculated for different "pore states" in alamethicin. ${ }^{9}$ Thus QDs appear to behave in a manner similar to alamethicin, aggregating to form pores capable of allowing ions to flow through the lipid bilayer. While an alamethicin pore is formed by peptide helices, and acts as a selectivity filter for most cations, ${ }^{10}$ a QD formed pore is not governed by helices and should therefore be nonselective.

Our demonstration that QDs elicit current bursts in synthetic membranes suggests that they could also do this in excitable cells such as neurons and muscles, or in any cell or organism with a bilayer membrane having an electrical potential (e.g., Paramecium, Saccharomyces cerevisiae, etc.). It would be of great utility to understand how QDs behave in vivo in excitable cells, especially because their use in tissue imaging is becoming widespread.

In summary, we have demonstrated that three different QDs elicit current fluctuations in artificial lipid bilayers. Our results show that these current bursts are voltage dependent and are due to the presence of a large permanent dipole moment of the QDs, which results in insertion into the lipid bilayer in the presence of an electric field. We believe that current fluctuations are due to formation of QD aggregates leading to pore formation as well as single dots breaking through the membrane leading to smaller current peaks. A collective event of such disruptions of the membrane would result in the pattern of current fluctuations we observe. Studying the gating mechanisms will be the next challenge.

The authors acknowledge support by a Vilas Associate award from the University of Wisconsin-Madison and the Office of Naval Research (ONR) (N00014-99-1-0717). The authors would like to thank Dr. Meyer B. Jackson (Department of Physiology, University of Wisconsin-Madison), and Ryan C. Toonen for their helpful suggestions.

${ }^{1}$ P. Alivisatos, Nat. Biotechnol. 22, 47 (2004).

${ }^{2}$ J. O. Winter, T. Y. Liu, B. A. Korgel, and C. E. Schmidt, Adv. Mater. (Weinheim, Ger.) 13, 1673 (2001).

${ }^{3}$ R. Coronado, Annu. Rev. Biophys. Biophys. Chem. 15, 259 (1986).

${ }^{4}$ P. Mueller and D. O. Rudin, Nature (London) 217, 713 (1968).

${ }^{5}$ M. Eisenberg, J. E. Hall, and C. A. Mead, J. Membr. Biol. 14, 143 (1973).

${ }^{6}$ R. Yantorno, S. Takashima, and P. Mueller, Biophys. J. 38, 105 (1982).

${ }^{7}$ G. Schwarz and P. Savko, Biophys. J. 39, 211 (1982).

${ }^{8}$ M. Shim and P. Guyot-Sionnest, J. Chem. Phys. 111, 6955 (1999).

${ }^{9}$ W. Hanke and G. Boheim, Biochim. Biophys. Acta 596, 456 (1980).

${ }^{10}$ R. O. Fox, Jr. and F. M. Richards, Nature (London) 300, 325 (1982). 